\title{
Analysis of the COL17A1 in non-Herlitz junctional epidermolysis bullosa and amelogenesis imperfecta
}

\author{
HIROYUKI NAKAMURA ${ }^{1}$, DAISUKE SAWAMURA ${ }^{1}$, MAKI GOTO ${ }^{1}$, HIDEKI NAKAMURA ${ }^{1}$, \\ MIYUKI KIDA ${ }^{2}$, TADASHI ARIGA ${ }^{2}$, YUKIO SAKIYAMA ${ }^{2}$, KOKI TOMIZAWA ${ }^{3}$, \\ HIROSHI MITSUI ${ }^{4}$, KUNIHIKO TAMAKI ${ }^{4}$ and HIROSHI SHIMIZU ${ }^{1}$ \\ ${ }^{1}$ Department of Dermatology, ${ }^{2}$ Research Group of Human Gene Therapy, Hokkaido University \\ Graduate School of Medicine, Sapporo 060-8638; ${ }^{3}$ Department of Dermatology, Ebetsu City Hospital, \\ Hokkaido; ${ }^{4}$ Department of Dermatology, Faculty of Medicine, University of Tokyo, Tokyo, Japan
}

Received January 31, 2006; Accepted March 27, 2006

\begin{abstract}
Non-Herlitz junctional epidermolysis bullosa (nHJEB) disease manifests with skin blistering, atrophy and tooth enamel hypoplasia. The majority of patients with $\mathrm{nH}-\mathrm{JEB}$ harbor mutations in COL17Al, the gene encoding type XVII collagen. Heterozygotes with a single COL17A1 mutation, $\mathrm{nH}-$ JEB defect carriers, may exhibit only enamel hypoplasia. In this study, to further elucidate COL17Al mutation phenotype/ genotype correlations, we examined two unrelated families with nH-JEB. Furthermore, we hypothesized that COL17Al mutations might underlie or worsen the enamel hypoplasia seen in amelogenesis imperfecta (AI) patients that are characterized by defects in tooth enamel formation without other systemic manifestations. We therefore conducted COL17A1 mutational analysis in three patients from two AI families. One nH-JEB patient showed no COL17Al expression and was a compound heterozygote for the novel premature termination codon (PTC) mutations 1285delA and Q1387X. In addition, reduced COL17Al expression was found in a second nH-JEB patient who was homozygous for the novel PTC mutation 4335delC, the most carboxyl terminal PTC mutation thus far identified. Due to nonsense mediated mRNA decay, the position of these PTC mutations is thought not to influence the effect of COL17Al transcript loss and hence the severity of the $\mathrm{nH}-$ JEB phenotype. This study is the first to suggest that type XVII collagen carboxyl PTC mutations lead to restoration of
\end{abstract}

Correspondence to: Dr Daisuke Sawamura, Department of Dermatology, Hokkaido University Graduate School of Medicine, North 15 West 7, Kita-ku, Sapporo 060-8638, Japan

E-mail: smartdai@med.hokudai.ac.jp

Abbreviations: AI, amelogenesis imperfecta; $\mathrm{nH}-\mathrm{JEB}$, non-Herlitz junctional epidermolysis bullosa; PCR, polymerase chain reaction; PTC, premature termination codon

Key words: type XVII collagen, blistering, enamel hypoplasia, mutation truncated polypeptide expression and to a milder clinical disease severity in $\mathrm{nH}-\mathrm{JEB}$. Conversely, we failed to detect any pathogenic COL17Al defects in AI patients, in either exon or within the intron-exon borders of AI patients. This study furthers the understanding of mutations in COL17Al causing $\mathrm{nH}-\mathrm{JEB}$, and clearly demonstrates that the mechanism of enamel hypoplasia differs between nH-JEB and AI diseases.

\section{Introduction}

Type XVII collagen, $180-\mathrm{kDa}$ bullous pemphigoid antigen is a transmembrane glycoprotein expressed in stratified and other complex epithelia, such as skin, mucous membranes, and the eye $(1,2)$. Type XVII collagen was initially identified as a major autoantibody target in bullous pemphigoid whereas mutations in the gene encoding this protein have been shown to cause junctional epidermolysis bullosa (JEB) (3). These facts indicate that type XVII collagen plays a crucial role in dermoepidermal attachment and hemidesmosome stability within the basement membrane zone.

JEB comprises a clinically and genetically heterogeneous group of disorders with tissue separation occurring within the lamina lucida of the epidermal basement membrane. To date, two major clinical variants of this subtype have been described: the moderately severe non-Herlitz JEB subtype (nH-JEB) characterized by life-long blistering and a non-lethal course; and the Herlitz type of JEB, associated with more severe and extensive blistering and a high perinatal mortality (4-6).

$\mathrm{nH}-J E B$ is characterized by several integument manifestations, including: blistering with skin atrophy, diffuse alopecia with loss of some eyelashes, eyebrows and pubic hair, as well as nail dystrophy and dental abnormalities. Recent studies showed that the gene encoding type XVII collagen (COL17A1) contains mutations in the majority of the patients with nH-JEB (6-14). However, the precise pathogenic mechanisms of all these various skin symptoms have yet to be elucidated.

Amelogenesis imperfecta (AI) is a rare heterogeneous group of genetic disorders characterized by defects in tooth enamel formation in the absence of any generalized or systemic 


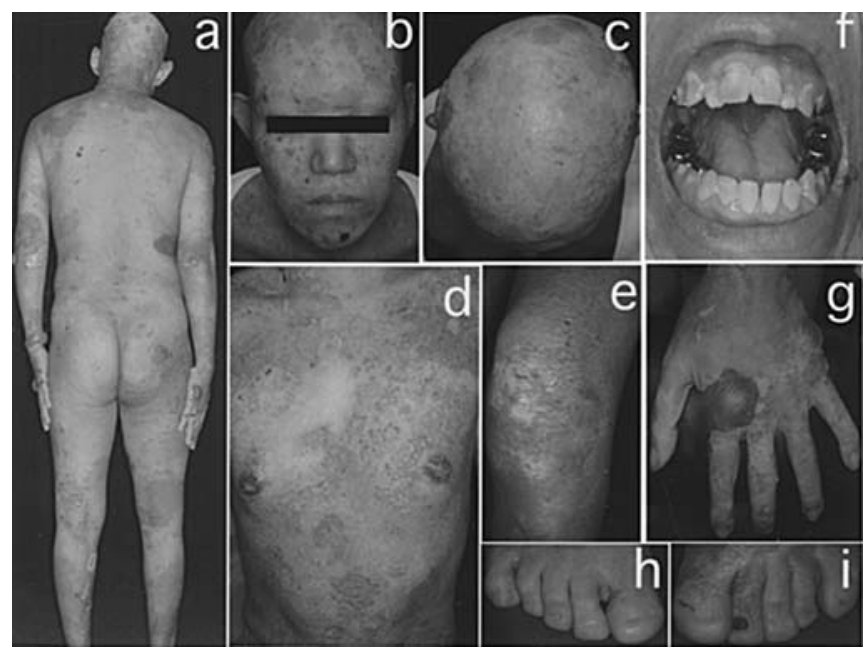

Figure 1. Clinical features of patient 1. There were many erosions and hyperpigmented or depigmented areas over his whole body (a), face (b), scalp (c) and trunk (d). Marked atrophic skin was found on his right knee (e). All his teeth showed abnormal enamel, with pitting and widespread dental caries (f). Large hemorrhagic blisters were seen on the left hand (g). Aonychia is seen on all the toenails and some fingernails (h and i).

diseases $(15,16)$. Significant progress has been made in determining the genetic background underlying AI. Mutations in the enamelin gene cause autosomal-inherited forms of AI, while $\mathrm{X}$-linked AI results from mutations in the amelogenin. Also, kallikrein-4, MMP-20 and DLX-gene have been shown to be causative genes in a limited number of cases. Congenital enamel hypoplasia and the resulting tooth pitting associated with severe dental caries are also characteristic features of nH-JEB. Particular COL17Al glycine substitution mutations can lead to enamel hypoplasia without skin blistering only when combined with a normal allele (9).

This study examined COL17Al mutations in two unrelated families with nH-JEB. Furthermore, to examine any possible correlation between enamel hypoplasia and nucleotide changes of COL17A1, we also searched for COL17A1 mutations in three patients from two distinct families with amelogenesis imperfecta (AI).

\section{Materials and methods}

\section{Families with $n H$-JEB}

Patient 1. A proband was a 21-year-old male from family 1 . He was the offspring of healthy nonconsanguinous parents and his elder brother was clinically unaffected. The patient had suffered recurrent blistering over his entire body since birth and had then suffered hair loss, dental problems, and dystrophic nails since infancy. Upon clinical examination (Fig. 1), bullae were seen on his extremities, abdomen, face, neck, and head, and there were depigmented or hyperpigmented skin lesions at recurrent sites of blistering without the presence of scars. The patient was bald, with an absence of eyelashes, eyebrows, pubic and axillary hair. His fingernails and toenails were dystrophic or missing. Marked hypoplasty of his permanent dentition was seen.

Patient 2. The second proband was a 41-year-old male from family 2 . He was the offspring of healthy parents and there

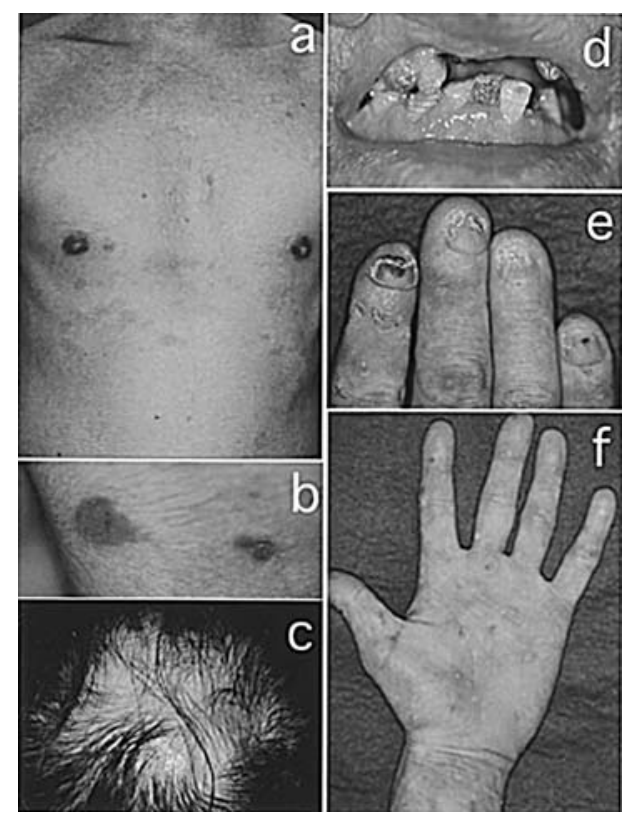

Figure 2. Clinical appearance of patient 2. There are several erosions and light hyperpigmentation on the trunk ( $a$ and $b$ ). On the parietal vertex of the scalp, there was focal non-scarring alopecia (c). Marked hypoplasty of the permanent dentition and dental caries were present (d), together with dystrophy of his fingernails (e), and small bullae (f) on his left palm were also seen.

was no history of consanguinity. No other family member had relevant skin manifestations. The patient had suffered from skin fragility over his whole body since birth. His hair growth was normal, but blisters and erosions had occurred on the scalp that led to patches of atrophic alopecia. The blisters healed without scars but some atrophy and hyperpigmentation had taken place. Upon clinical examination (Fig. 2), several bullae or erosions were seen on his extremities. Depigmentation or hyperpigmentation were present at recurrent sites of blistering without the presence of scars. Some of his fingernails were dystrophic or missing. There were irregular-shaped areas of alopecia on the parietal scalp, but his eyebrows, eyelashes, pubic and axillary hair were present. His teeth showed fragile enamel and had a tendency to exhibit dental caries. He also demonstrated marked focal hyperkeratosis on his palms and soles. We have previously published this patient highlighting his unusual hyperkeratotic lesions (17).

Families with amelogenesis imperfecta (AI). We investigated 3 patients diagnosed with an autosomal-dominant form of AI. Two patients were related, a father (patient 3) and son (patient 4). Their clinical findings were published elsewhere (18). Mutation analysis of candidate genes revealed they harboured heterozygous single $\mathrm{G}$ deletion within a series of 7 residues at the exon 9-intron 9 boundary of the enamelin gene (18). The other AI patient (patient 5) was a 22-year-old male and his family members were clinically unaffected. He was normally delivered and, at 2 years of age, abnormal teeth were first recognized (Fig. 3). All affected patients exhibited enamel hypoplasia in both the deciduous and permanent dentition without any other general complications, including blistering of the skin or oral mucosa. 


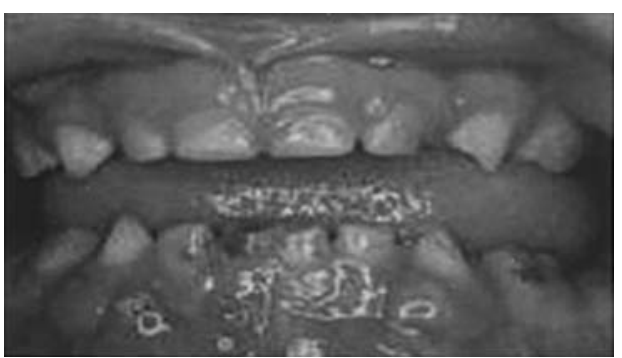

Figure 3. Clinical appearance of patient 5 with amelogenesis imperfecta. The AI patient exhibited enamel hypoplasia in both the deciduous and permanent dentition without any additional general complications, including skin blistering.

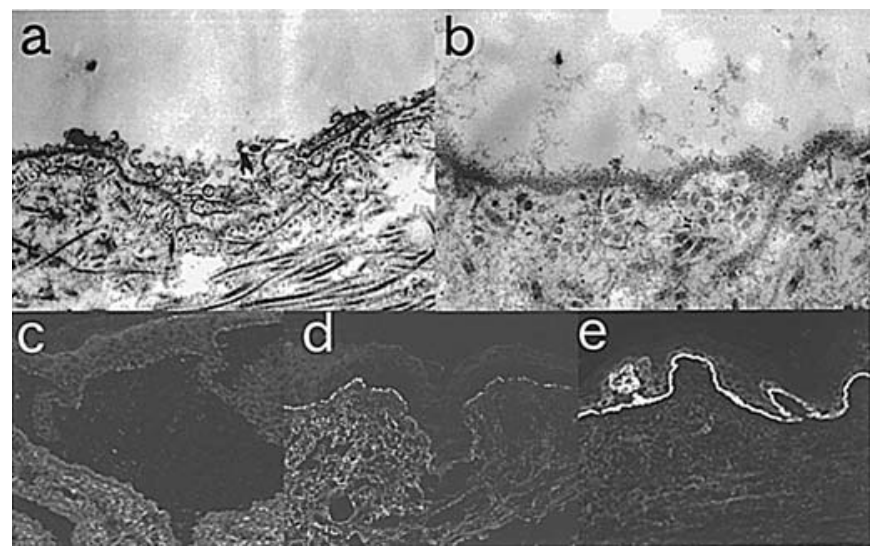

Figure 4. Ultrastructural and immunohistochemical analyses. Blistered skin from patients 1 (a) and 2 (b) showed tissue ultrastructural separation within the lamina lucida. Immunoreactivity of type XVII collagen was totally absent in patient 1 (c) while patient 2 showed decreased intermittent immunoreactivity at the base of the blister. Normal skin displayed bright, linear and continuous staining along the dermal-epidermal junction (e).

Immunohistochemical and ultrastructural analyses. Direct immunofluorescence analysis was performed using cryostat skin sections from patients as described previously (19). The monoclonal HDD 20 against human type XVII collagen was a kind gift from Dr Sonnenberg, The Netherlands Cancer Institute and used for these experiments. For electron microscopic examination, skin specimens from patients 1 and 2 were fixed in $5 \%$ glutaraldehyde and postfixed in $1 \%$ osmium tetroxide, stained en-block in uranyl acetate. They were dehydrated in a graded series of ethanol solutions, and then embedded in Araldite 6005. Ultrathin sections were cut, stained with uranyl acetate and lead citrate. The sections were examined with a transmission electron microscope (H-7100; Hitachi, Tokyo, Japan) at $75 \mathrm{kv}$.

Mutation analysis of COL17A1. Genomic DNA was isolated from peripheral lymphocytes from the patients and their families using standard procedures. The mutation detection strategy was performed after polymerase chain reaction (PCR) amplification of all exons including the intron-exon borders, followed by direct automated nucleotide sequencing using the BigDye terminator system (Applied Biosystems, Foster City, CA). The genomic DNA nucleotides were numbered based on the previous sequence information (GenBank

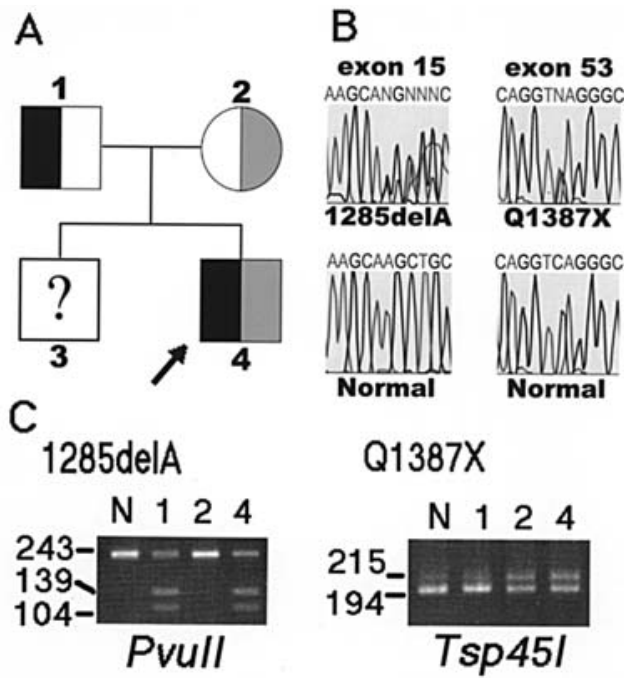

Figure 5. Identification of COL17A1 mutations in patient 1 with non-Herlitz junctional epidermolysis bullosa. A: family pedigree; black areas, the deletion mutation 1285delA; gray areas, the nonsense mutation Q1387X. We could not obtain samples from the brother (3). B: Direct nucleotide sequencing of exon 15 in the father (1) and the proband patient (4) revealed a heterozygous 1-bp deletion of nucleotide A at position c.1285, whereas exon 53 of the mother (2) and the patient (4) showed a heterozygous C-to-T transition at position c.4264, which changes a glutamine 1387 to a stop codon (Q1387X). C: 1285delA generated the PvuII site within the 243-bp PCR product (g.37 to g.279: GenBank accession no.: U76578), which was digested to 139-bp and 104-bp fragments. Q1387 abolished the Tsp45I site of the 215-bp PCR product (g.-77 to g.128: U76603), which failed to be digested to 194-bp and 21-bp fragments in control samples. N: normal individual.

accession no.: U76564-U76604). PCR amplification of all 56 exons of the COL17Al gene was carried out as described previously (11).

\section{Results}

Immunohistochemical and ultrastructural results. Electron microscopic examination of the skin samples from patients 1 and 2 revealed that the tissue separation was localized within the lamina lucida (Fig. 4a and b). The lamina densa was well formed at the base of the split whereas the basal cell membrane was present at the roof of the separation opposing the lamina densa. The ultrastructural findings were consistent with these cases being classified as JEB. Immunoreactivity using the monoclonal antibody HDD20 against type XVII collagen was totally absent in patient 1 while patient 2 showed reduced intermittent or granular immunoreactivity along the dermal-epidermal junction (Fig. 3c and d). Normal skin demonstrated bright, linear and continuous staining of the dermal-epidermal junction (Fig. 3e).

Detection of COL17A1 mutations. Mutation screening included direct PCR amplification of COL17A1 exons from genomic DNA. Patient 1 was compound heterozygous for a deletion of the base A at position c.1285 in exon 15 and for a C-to-T transition at position c.4264 in exon 53 (Fig. 5). The $1285 \mathrm{del}$ A deletion led to a premature termination codon (PTC) 26-bp downstream from the site of deletion, and the $\mathrm{C} \rightarrow \mathrm{T}$ transition caused the conversion of codon CAG (Gln) to a PTC at amino acid 1387 and was designated Q1387X. 


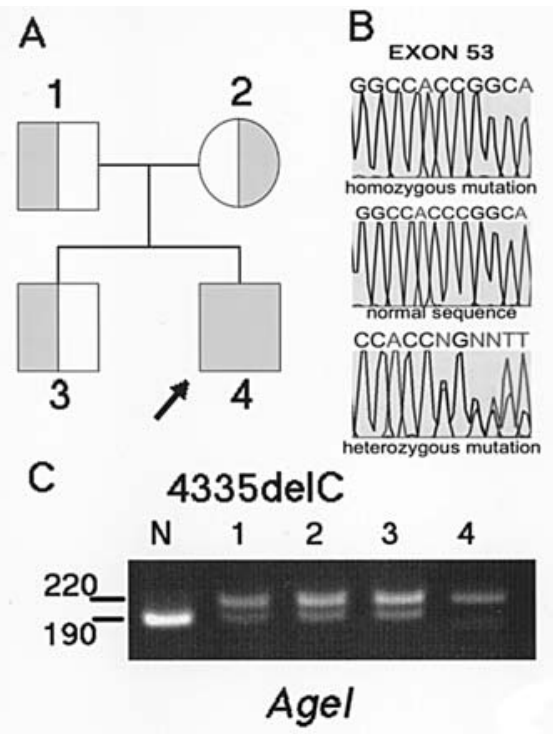

Figure 6. Identification of COL17A1 mutations in patient 2 with non-Herlitz junctional epidermolysis bullosa. A: family pedigree: the gray area: the deletion mutation $4335 \mathrm{delC}$. B: direct nucleotide sequencing of exon 53 revealed homozygous 1-base deletions of a $\mathrm{C}$ nucleotide at c.4335 in the patient (4), and a heterogeneous mutation in the other family members $(1,2$, 3). C: 4335 delC abolished the AgeI site of the 210 PCR product (g.-10 to g.200: GenBank accession no.: U76603), which was not digested to $190 \mathrm{bp}$ and $20 \mathrm{bp}$ fragments. N: normal individual.

Nucleotide sequencing showed that the 1285 delA mutation was paternal and the Q1387X mutation was maternal. A DNA sample from the unaffected brother was not available. Patient 2 was homozygous for a deletion of $\mathrm{C}$ at position c.4335 in exon 53 (4335delC) that led to a PTC 35-bp down-stream from the deletion (Fig. 6). His father, mother, and older brother were all heterozygous for this mutation. The mutations were confirmed by restriction enzyme digestion (Figs. 5 and 6).

No significant nucleotide changes were noted in COL17A1 in AI patients. Analysis of all 56 exons of the COL17A1 gene by direct sequencing failed to uncover any mutations in patients 3, 4 and 5 except for common gene polymorphisms (data not shown).

\section{Discussion}

Herein, we report two nH-JEB patients with tissue separation within the lamina lucida. The patients showed common phenotypic attributes including generalized blistering, skin atrophy, diffuse alopecia, nail dystrophy and dental abnormalities. Patient 1 was compound heterozygote for a deletion mutation (1285delA) in the intracellular domain and a nonsense mutation (Q1387X) in the extracellular domain, both of which are novel. Patient 2 also harboured a novel homozygous deletion mutation 4335delC.

As far as we know, >60 mutations in the COL17Al gene have been reported thus far (20). The majority of the reported mutations in the COL17Al gene cause premature termination codons (PTC) as a result of either nonsense or small insertion/ deletion mutations. In all these cases, an absence of immunofluorescence staining was found in the patients' skin for type
XVII collagen. Thus, nonsense mediated decay of the abnormal transcripts are responsible for the lack of type XVII collagen at both the mRNA and protein level in these patients $(3,10)$. In this respect, the position of the nonsense mutations might not be associated with the severity of nH-JEB phenotype, since any nonsense mutations will lead to a drastically reduced level of both collagen XVII mRNA and protein levels.

Although the clinical features of patients 1 and 2 were typical for $\mathrm{nH}-\mathrm{JEB}$, the second patient demonstrated fewer blisters and erosion, and milder alopecia with some normal hair growth compared to patient 1 . Furthermore, patient 2 showed discontinuous immunoreactivity along dermalepidermal junction, which indicated expression of type XVII collagen. The homozygous $4335 \mathrm{delC}$ mutation in patient 2 was the most carboxyl terminal homozygous mutation of all previous COL17A mutations (20). The literature concerning nH-JEB cases with homozygous nonsense mutations suggests that the position of mutations on either the collagenous or the noncollagenous domains might affect the severity of the nH-JEB phenotype (6). In this study, the most carboxyl terminal homozygous mutation showed a milder phenotype. This suggests that more carboxyl PTC mutations in the type XVII collagen polypeptide may lead to a restoration of truncated polypeptide expression, resulting in the milder clinical severity.

Enamel hypoplasia and the resulting tooth pitting associated with severe caries, are also characteristic features of nH-JEB. As previously reported, both the ligand laminin 5 and its receptor in skin, the $\alpha 6 \beta 4$ integrin are thought to be important in tooth morphogenesis (21-23). Mutations in the genes encoding type XVII collagen, laminin 5 and $\alpha 6 \beta 4$ integrins result in JEB, and some interaction between those molecules has been suggested. Furthermore, the dental abnormality may also be caused by the hemizygousity of a dominant negative COL17Al missense mutation G627V (9). Therefore, we hypothesized that any mutations in the COL17Al gene might be associated with a genetic enamel hypoplasia and severely disrupted enamel formation. Thus, we have searched for nucleotide changes in the COL7AI gene of patients with amelogenesis imperfecta (AI).

AI comprises a clinical and genetically heterogeneous group of inherited defects with dental enamel formation but without the significant involvements of other organs and this disease group can be classified into a further 14 phenotypic subtypes. The process of dental enamel formation involves proteins secreted by ameloblast cells, which have an epithelial origin. To date, mutations have been identified in the enamelin gene that causes autosomal-inherited forms of AI, while Xlinked AI results from mutations in the amelogenin. In addition, defects in kallikrein-4, MMP-20 and DLX-gene can also underlie AI genetic mutations in some cases $(16,18,24)$. This study analyzed, by direct sequencing, all 56 exons of the COL17A1 gene in 3 AI patients. As a result, we failed to identify any association between $\mathrm{AI}$ and collagen XVII and no significant COL17AI nucleotide changes were present in any AI cases.

In summary, we have examined COL17Al genes in $2 \mathrm{nH}-$ JEB families and 2 AI families with enamel hypoplasia. We demonstrated three novel mutations in $\mathrm{nH}-\mathrm{JEB}$ families, but no significant nucleotide changes in COL17A1 in AI pedigrees. 
This suggests that the mechanisms causing enamel hypoplasia are different between $\mathrm{nH}-\mathrm{JEB}$ and AI.

\section{Acknowledgements}

We wish to thank James R. McMillan for his critical reading of and comments concerning this study. This work was supported in part by Grants-in-Aid from the Ministry of Education, Science, Sports, and Culture of Japan (15390336 to H.S., and 15390337 to D.S.) and by grants from the Ministry of Health of Japan (H16-Nanji-05 to H.S.).

\section{References}

1. Zillikens D and Giudice GJ: BP180/type XVII collagen: its role in acquired and inherited disorders or the dermal-epidermal junction. Arch Dermatol Res 291: 187-194, 1999.

2. Borradori L and Sonnenberg A: Structure and function of hemidesmosomes: more than simple adhesion complexes. J Invest Dermatol 112: 411-418, 1999.

3. McGrath JA, Gatalica B, Christiano AM, et al: Mutations in the 180-kD bullous pemphigoid antigen (TYPE XVII COLLAGEN), a hemidesmosomal transmembrane collagen $(C O L 17 A 1)$, in generalized atrophic benign epidermolysis bullosa. Nat Genet 11: 83-86,1995.

4. Hashimoto I, Schnyder UW and Anton-Lamprecht I: Epidermolysis bullosa hereditaria with junctional blistering in an adult. Dermatologica 152: 72-86, 1976.

5. Hintner $\mathrm{H}$ and Wolff $\mathrm{K}$ : Generalized atrophic benign epidermolysis bullosa. Arch Dermatol 118: 375-384, 1982.

6. Schumann H, Hammami-Hauasli N, Pulkkinen L, et al: Three novel homozygous point mutations and a new polymorphism in the COL17A1 gene: relation to biological and clinical phenotypes of junctional epidermolysis bullosa. Am J Hum Genet 60: 1344-1353, 1997.

7. Floeth M, Fiedorowicz J, Schacke H, et al: Novel homozygous and compound heterozygous COL17A1 mutations associated with junctional epidermolysis bullosa. J Invest Dermatol 111: 528-533, 1998.

8. McGrath JA, Darlig T, Gatalica B, et al: A homozygous deletion mutation in the gene encoding the $180-\mathrm{kDa}$ bullous pemphigoid antigen (TYPE XVII COLLAGEN) in a family with generalized atrophic benign epidermolysis bullosa. J Invest Dermatol 106: 771-774, 1996.

9. McGrath JA, Gatalica B, Li K, et al: Compound heterozygosity for a dominant glycine substitution and a recessive internal duplication mutation in the type XVII collagen gene results in junctional epidermolysis bullosa and abnormal dentition. Am J Pathol 148: 1787-1796, 1996

10. Darling TN, McGrath JA, Yee C, et al: Premature termination codons are present on both alleles of the bullous pemphigoid antigen 2/type XVII collagen gene in five Austrian families generalized atrophic benign epidermolysis bullosa. J Invest Dermatol 108: 463-468, 1997.
11. Gatalica B, Pulkkinen L, Li K, et al: Cloning of the human type XVII collagen gene (COL17A1), and detection of novel mutations in generalized atrophic benign epidermolysis bullosa. Am J Hum Genet 60: 352-65, 1997.

12. Jonkman MF, De Jong MCJM, Heeres K, et al: Generalezed atrophic benign epidermolysis bullosa. Either 180-kd bullous pemphigoid antigen or laminin-5 deficiency. Arch Dermatol 132: $145-150,1996$.

13. Scheffer H, Stulp RP, Verlind E, et al: Implications of intragenic marker homozygosity and haplotype sharing in a rare autosomal recessive disorder: the example of the collagen type XVII (COL17Al) locus in generalized atrophic benign epidermolysis bullosa. Hum Genet 100: 230-235, 1997.

14. Shimizu H, Takizawa Y, Pulkkinen L, et al: The $97 \mathrm{kDa}$ linear IgA bullous dermatosis antigen is not expressed in a patient with generalized atrophic benign epidermolysis bullosa with a novel homozygous G258X mutation in COL17A1. J Invest Dermatol 111: 887-892, 1998.

15. Witkop CJJ: Amelogenesis imperfecta, dentinogenesis imperfecta and dentin dysplasia revisited: problems in classification. J Oral Pathol 17: 547-553, 1998.

16. Stephanopoulos G, Garefalaki ME and Lyroudia K: Genes and related proteins involved in amelogenesis imperfecta. J Dent Res 84: 1117-1126, 2005

17. Mitsui H, Watanabe T, Komine M, et al: Focal palmoplantar callosities in non-Herlitz junctional epidermolysis bullosa. J Am Acad Dermatol 52: 371-373, 2005.

18. Kida M, Ariga T, Shirakawa T and Sakiyama Y: Autosomaldominant hypoplastic form of amelogenesis imperfecta caused by an enamelin gene mutation at the exon-intron boundary. $\mathbf{J}$ Dent Res 81: 738-742, 2002.

19. Shimizu H, Suzumori K, Hatta N and Nishikawa T: Absence of detectable $\alpha 6$ integrin in pyloric atresia-junctional epidermolysis bullosa syndrome and its application for prenatal diagnosis in a family at risk for recurrence. Arch Dermatol 132: 919-925, 1996.

20. Bauer JW and Lanschuetzer C: Type XVII collagen gene mutations in junctional epidermolysis bullosa and prospects for gene therapy. Clin Exp Dermatol 28: 53-60, 2003.

21. Aberdam D, Aguzzi A, Baudoin C, Galliano MF, Ortonne JP and Meneguzzi G: Developmental expression of nicein adhesion protein (laminin-5) subunits suggests multiple morphogenetic roles. Cell Adhes Commun 2: 115-129, 1994.

22. Thesleff I: Differentiation of ameloblasts and its regulation by epithelial-mesenchymal interactions. In: Dental Enamel: Formation to Destruction. Robinson C, Kirkham J and Shore RC (eds). CRC Press, Boca Raton, pp1-22, 1995.

23. Skobe Z, Stern DN and Prostak KS: The cell biology of amelogenesis. In: Dental Enamel: Formation to Destruction. Robinson C, Kirkham J and Shore RC (eds). CRC Press, Boca Raton, pp23-57, 1995.

24. Hu JC and Yamakoshi Y: Enamelin and autosomal-dominant amelogenesis imperfecta. Crit Rev Oral Biol Med 14: 387-398, 2003. 